\title{
Utilizing Smart City Cyber-physical Infrastructure for Tracking and Monitoring Pandemics like COVID-19 with the ICCC as the Nerve Centre
}

\author{
DIBYENDU ROY, NITIN AGARWAL, AMITAVA MUKHERJEE, JITENDRA ANGNE, \\ ANEES FARHAN, DEVEN SORATHIYA, R. DEVARAJAN, and \\ MANUKUMAR VELAYUDHAN NAIR, Hewlett Packard Enterprises
}

\begin{abstract}
Managing the ongoing COVID-19 (aka Coronavirus) pandemic has presented both challenges and new opportunities for urban local body administrators. With the Indian government's Smart City mission taking firm roots in some of the Indian cities, the authors share their learnings and experiences of how a Smart City Integrated Command and Control Centre (ICCC) can be extended to become the nerve centre of pandemic-related operations and management, leveraging the Smart City IoT infrastructure such as surveillance cameras for monitoring and enforcement. The authors are of the opinion that the lessons learned and experiences gained from these cities are extremely valuable and can easily be replicated in other cities in a relatively short time period, thus providing a standard and uniform method across the nation for handling epidemics in the future.
\end{abstract}

CCS Concepts: • Applied computing $\rightarrow$ Enterprise applications;

Additional Key Words and Phrases: ICCC - Integrated Command and Control Centre, ICMR - Indian Council of Medical Research, ICT - Information and Communication Technologies, MIS - Management Information System, SOP - Standard Operating Procedures, SMS - Short Messaging Service, VMS - Video Management System, VA - Video Analytics

\section{ACM Reference format:}

Dibyendu Roy, Nitin Agarwal, Amitava Mukherjee, Jitendra Angne, Anees Farhan, Deven Sorathiya, R. Devarajan, and Manukumar Velayudhan Nair. 2020. Utilizing Smart City Cyber-physical Infrastructure for Tracking and Monitoring Pandemics like COVID-19 with the ICCC as the Nerve Centre. Digit. Gov.: Res. Pract. 2, 1, Article 8 (November 2020), 8 pages.

https://doi.org/10.1145/3428124

\section{INTRODUCTION}

Due to the COVID-19 Pandemic, the government of India ordered a nationwide lockdown, limiting movement of the entire 1.3 billion population as a preventive measure [1]. The lockdown was implemented to contain the spread, and tests were being conducted on symptomatic and infected patients and citizens with co-morbidities. Testing guidelines were followed as advised by the Indian Council of Medical Research (ICMR), Ministry of

Authors' addresses: D. Roy, N. Agarwal, A. Mukherjee, J. Angne, A. Farhan, D. Sorathiya, R. Devarajan, and M. V. Nair, Hewlett Packard Enterprises, Salarpuria Arena, Adugodi, Hosur Main Road, Bangalore, Pin code: 560093, India; emails: \{dibyendu.roy, nitin.agarwal, amitava.mukherjee, jitendra.agne, anees.farhan, deven.sorathiya, r.devarajan, manu-kumar\}@hpe.com.

Permission to make digital or hard copies of all or part of this work for personal or classroom use is granted without fee provided that copies are not made or distributed for profit or commercial advantage and that copies bear this notice and the full citation on the first page. Copyrights for components of this work owned by others than ACM must be honored. Abstracting with credit is permitted. To copy otherwise, or republish, to post on servers or to redistribute to lists, requires prior specific permission and/or a fee. Request permissions from permissions@acm.org. (c) 2020 Association for Computing Machinery. 2639-0175/2020/11-ART8 \$15.00

https://doi.org/10.1145/3428124 
Health and Family Welfare, Ministry of Home, and the respective state government agencies [2]. COVID-19 tests were being conducted at various government offices and approved private labs. Citizens who tested positive were advised institutional quarantine and isolation, but later on, due to a resource crunch, were home quarantined and monitored for their health conditions [3].

During lockdown, citizens were facing problems related to food purchase and difficulty in their travel for medical and other unavoidable personal emergencies. Outstation citizens who had travelled for work, business, education, and medical treatment faced not only the same difficulties but also were seeking authorities' assistance to return to their hometowns. Authorities quickly realised that it could not be addressed through manual means and started looking out for automated and Information and Communication Technologies (ICT) based solutions.

Lockdown, testing, quarantine, and monitoring measures are effectively implemented by various state governments through the state police and the urban local bodies like Municipal Corporations under the supervision of the State Health Department and ICMR [4].

Many municipal corporations in India have built Integrated Command Control Centres (ICCC) for integrated city operations, as part of their Smart City project initiatives along with Internet of Things (IoT; cyber-physical) systems to manage and monitor the city [5]. This infrastructure offers a city or the urban local bodies an excellent facility and a head-start in implementing ICT-assisted measures for monitoring effectiveness of lockdown, COVID-19 monitoring, and management by enabling effective coordination amongst various agencies, bringing them under one roof. Repurposed Smart City infrastructure swiftly created and implemented several use cases for automatic detection, reporting, and Standard Operating Procedure (SOP) executions, resulting in reduced human field exposure.

In this technical article, the authors discuss how select Indian cities have been enabled to leverage their existing Smart City infrastructure-the ICCC and the IoT devices like surveillance cameras-toward the following [6]:

-Lockdown tracking and monitoring, citizen support, and assistance $[4,7]$

-Quarantine Center surveillance and monitoring [3, 8]

- COVID-19 test assistance management and monitoring [2, 9]

\section{Lockdown Tracking and Monitoring, Citizen Support, and Assistance}

Lockdown restrictions for movement have not gone down well with the people, and incidents were reported of people breaking the lockdown measures and gathering in large numbers either due to negligence or ignorance.

With limited resources on the authorities' part that were already stretched to the hilt, they turned toward smarter and automated ways to detect crowds and gatherings, and to monitor people breaking the lockdown measures in public places.

Crowd-tracking use cases real-time scenarios and the associated process flow within the Data Exchange Server (DES) are depicted in Figure 1. Every connected video app works as a stand-alone micro service, which communicates with the DES for its operation and exchange of data, with its involved components as shown in the Figure 1 block diagram.

Smart City "City Surveillance" solution has installed 8-megapixel bullet type Closed Circuit Television (CCTV) cameras in many locations to capture and transmit live feed to the respective ICCC for monitoring law and order [7, 10]. They utilize the Video Management System (VMS) and real-time Video Analytics (VA) to detect such events. Authorities have defined SOPs for handling such events at the ICCC.

The Smart City team decided to use these camera installations for lockdown monitoring and crowd detection using the following steps (reference Figure 2).

Step 1. Identify potential and vulnerable locations of crowd gathering, such as marketplaces, petty shops, and so on where lockdown measures and social distancing norms have to be strictly followed.

Digital Government: Research and Practice, Vol. 2, No. 1, Article 8. Publication date: November 2020. 

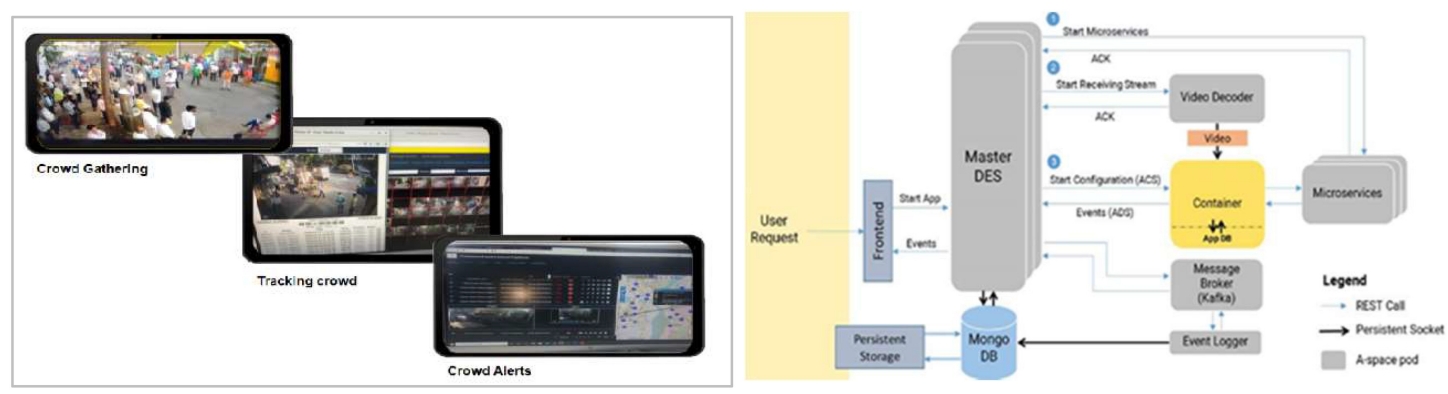

Fig. 1. Crowd tracking and associated execution flow DES diagram.

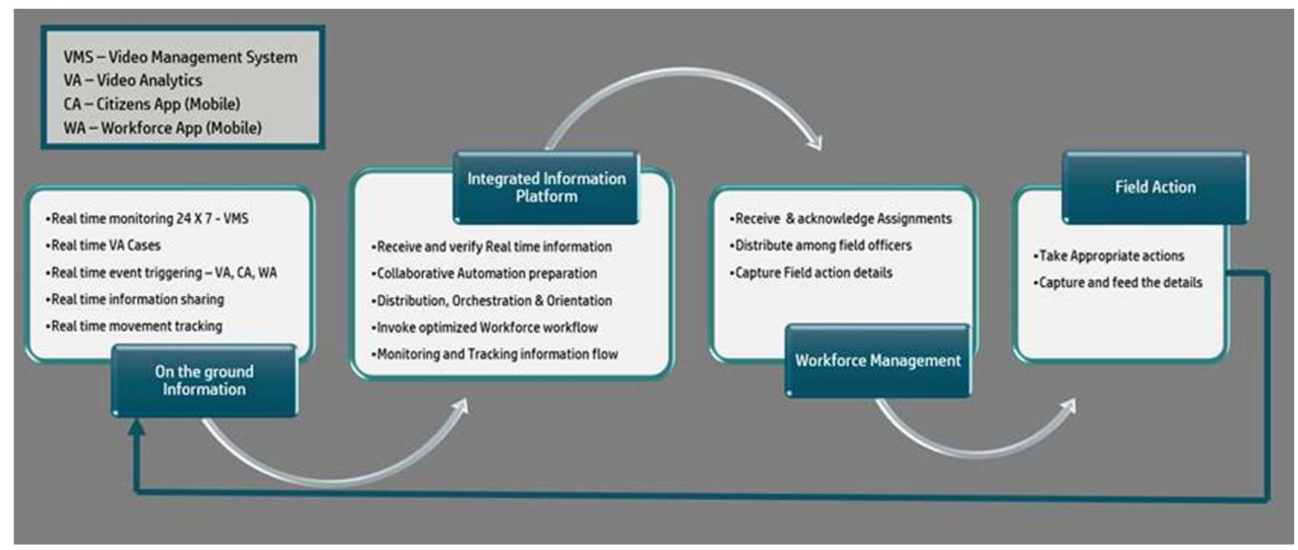

Fig. 2. Crowd detection, tracking, and alert generation process.

Step 2. If unavailable, install CCTV cameras in the above locations.

Step 3. Define the various threshold parameters within the video management and analytics solution for crowd detection, head counting, and generating an alarm; display the same at the ICCC along with the location details and time of the occurrence, if the threshold parameters are breached.

Step 4. Alert authorities monitoring these parameters immediately and dispatch the patrol teams or the mobile workforce to take appropriate actions.

This remote crowd detection monitoring and reporting helps authorities optimise their overstretched resources, take timely action, and reduce exposure risks. Implementation of this use case was with the sole objective of crowd detection alone, devoid of capturing and storing any photographs of the individuals so that any privacy intrusions can be completely avoided.

The technical architecture of the implemented solution is shown in Figure 3.

Another key initiative was to ask citizens to use the City Mobile App to report to authorities, such crowding incidents or incidents where social distancing norms are not followed, through a City Mobile App, providing the location details along with the video or snap of the scene to Smart City ICCC. This helped authorities to take timely action in areas where CCTV camera surveillance was not available.

During lockdown, authorities had to provide essential civic services on-demand over electronic channels. Civic services that required a citizen to visit the municipal offices before the pandemic and lockdown are now extended through the Citizen Mobile App to the citizens' doorsteps without them having to step outside the safety of their homes. This in turn reduced the need for citizens to venture out of their homes. Services were categorized into 


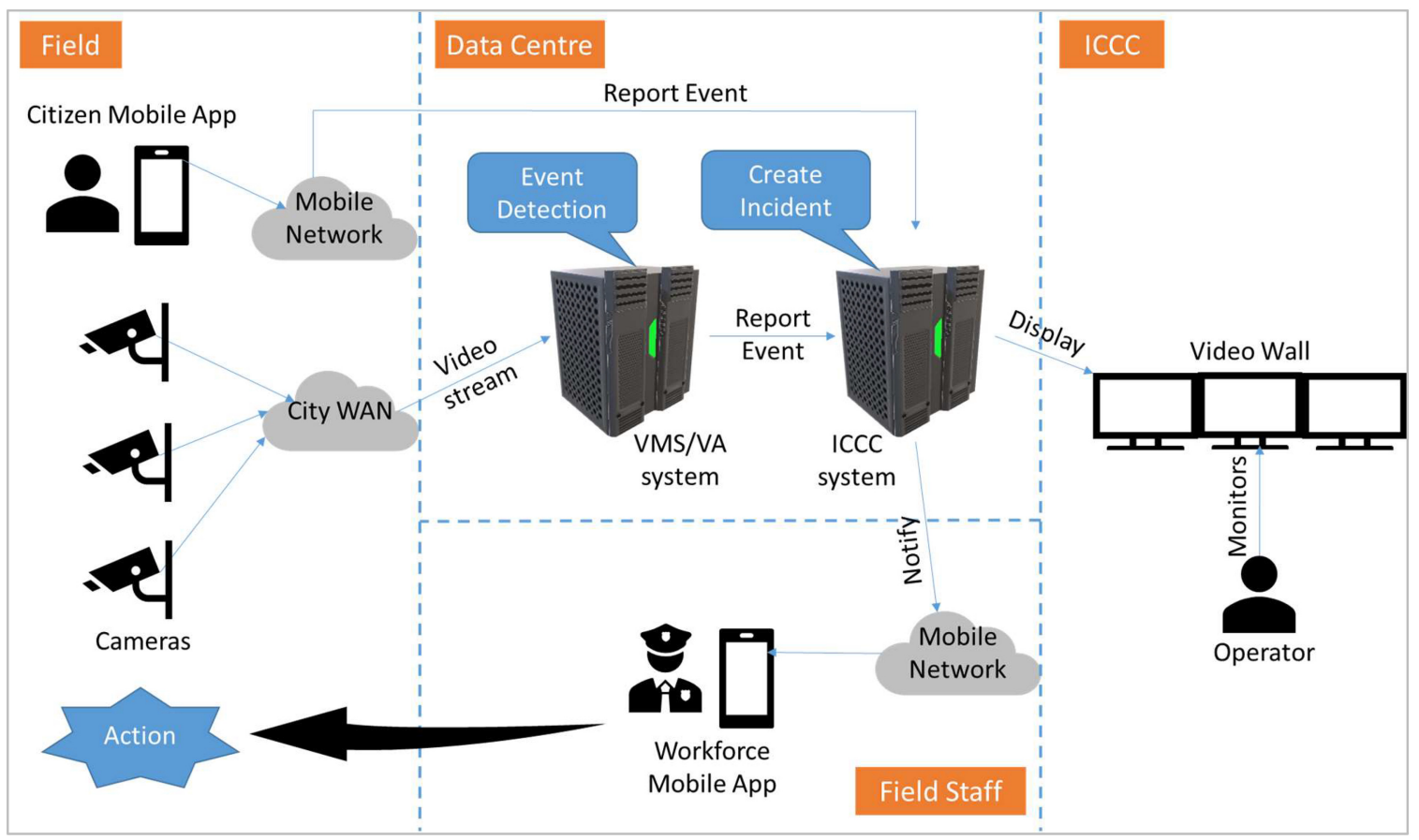

Fig. 3. Technical architecture of lockdown tracking and monitoring.

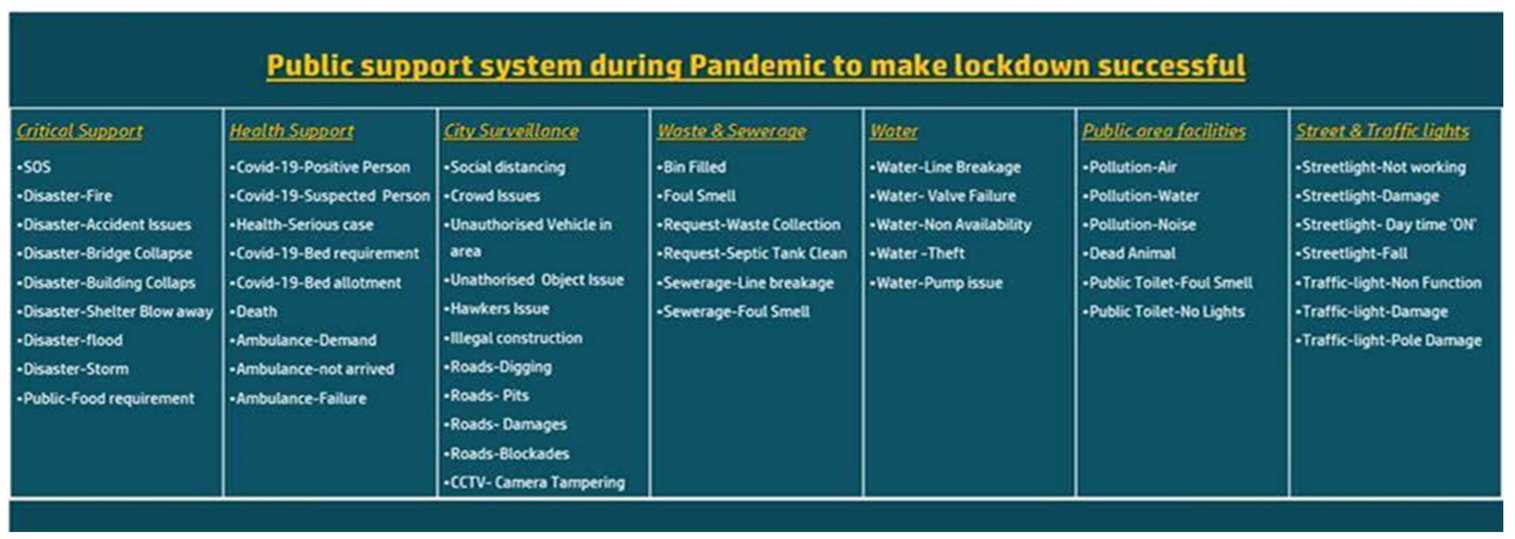

Fig. 4. Smart City offered public support system.

many buckets to raise their demands through the mobile App. The citizen demands were centrally managed at the Smart City ICCC platform. Field level officers and staff were provided with a Workforce Mobile App to have an automated communication platform to mobilise civic staff where required, thus optimising the workforce on the field and reducing their exposure risk.

The table in Figure 4 depicts the list of services that citizens could raise a request for. 


\section{Quarantine Centre Surveillance System}

As part of COVID-19 containment plans, authorities had established quarantine centers for COVID suspects, and domestic and international travellers. Manual surveillance measures became a challenge to authorities who looked at Smart City teams for automatic and ICT enabled measures for support.

Smart Cities used a CCTV camera-based facial recognition system to detect and report incidents of quarantine breach. The authorities were capturing the photograph of the quarantined citizens at the time of admission into the quarantine centre $[3,4,8,10]$.

It involved the following steps:

Step 1. Installing CCTV cameras (typical 8-megapixel bullet cameras) at strategic locations within the quarantine centres identified by the authorities. Cameras are positioned at strategic locations such as entry and exit points, driveways, or the vehicle parking space of the quarantine centre.

Step 2. Implementing an edge computing device for face recognition in the video stream.

Step 3. Periodically updating the edge computing device with the photograph of the quarantined citizens to be monitored and tracked.

Step 4. Monitoring the video stream centrally from these cameras from multiple quarantine centres through the edge computing device.

Step 5. Generating an alarm centrally at the ICCC if a quarantined person ventures out.

However, implementing face recognition for this task was not without challenges. As an overall safety measure, everyone was instructed to wear face masks, which added to the complexity and difficulty in identifying and detecting a citizen breaching the quarantine. The face recognition system was strengthened with capabilities to identify this person, from exposed facial region beyond the mask covered area.

This limited facial area based person detection was implemented by providing the system with multi-angle face photographs of the quarantined citizens for identifying various levels of unmasked facial area. This system has helped remotely manage the citizens' movements in the quarantine centres. In this use case, photographs of the detected person involved were used for the sole purpose of alerting the systems and authorities involved. Photographs were deleted after the COVID safety SoP was executed to ensure that no personal data was captured and stored.

Edge compute was implemented to quickly perform the analytics required for facial recognition, which enabled authorities to quickly act on any breaches. Applicable country guidelines for data sharing and privacy were followed strictly while deploying these use cases.

The technical architecture for this solution is shown below in Figure 5.

\section{COVID-19 Management Platform}

In addition to leveraging Smart City IoT infrastructure for lockdown monitoring, management, and quarantine surveillance, the authors implemented a solution for COVID-19 management, test assistance management, and monitoring in a different Smart City [5]. The solution supports a single window tracking of COVID-19 patients, identifies new suspected patients through first and secondary contact tracking, lab modules for updating test results, and hospitalisation records. Though it did not involve any IoT system, it leverages the Smart City ICCC as the nerve centre for COVD-19 management, test management, and reporting based on the symptoms experienced by citizens and manages the full life cycle of such cases. It also emphasises the need for a central test management and reporting.

Life cycle of a COVID-19 test includes (as mandated by the authorities) [11]:

Step 1. Identifying cases for COVID-19 tests, which typically happens when door-to-door surveys are conducted in hotspots or contaminated areas of the city, or when a citizen reports being unwell at designated hospitals.

Digital Government: Research and Practice, Vol. 2, No. 1, Article 8. Publication date: November 2020. 


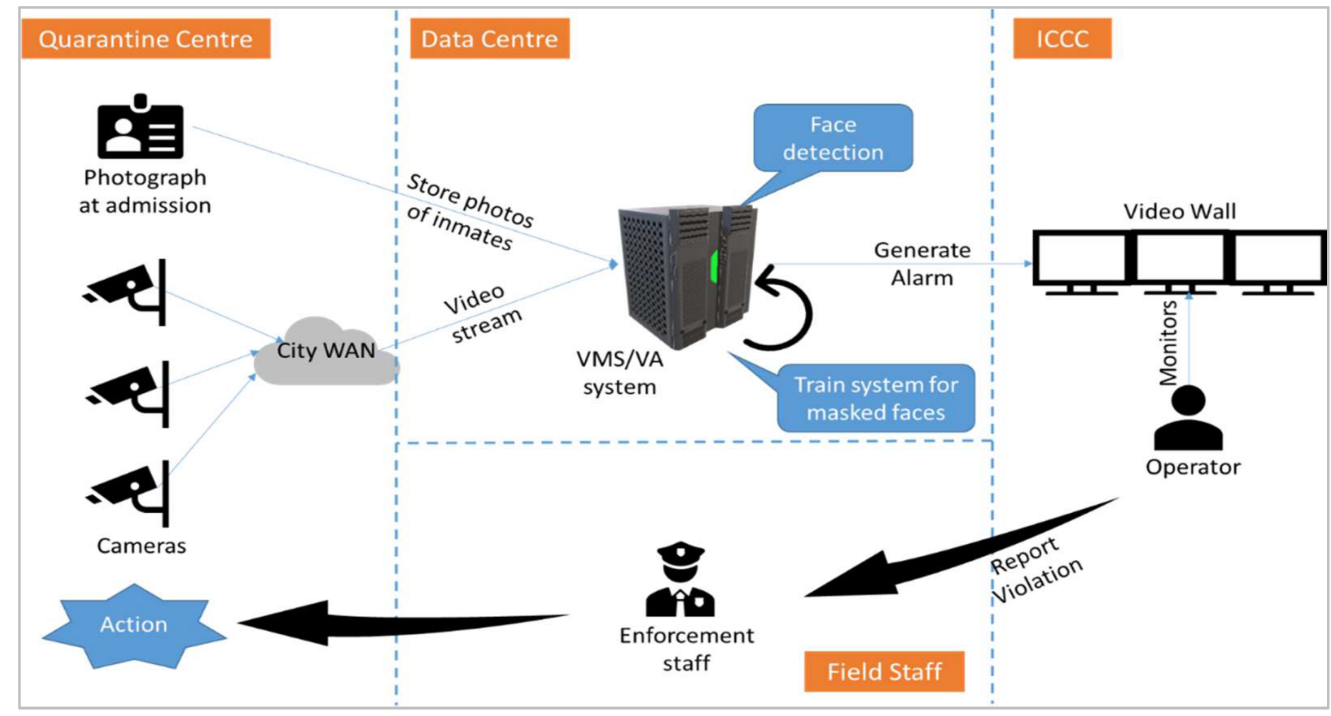

Fig. 5. Technical architecture for quarantine monitoring.

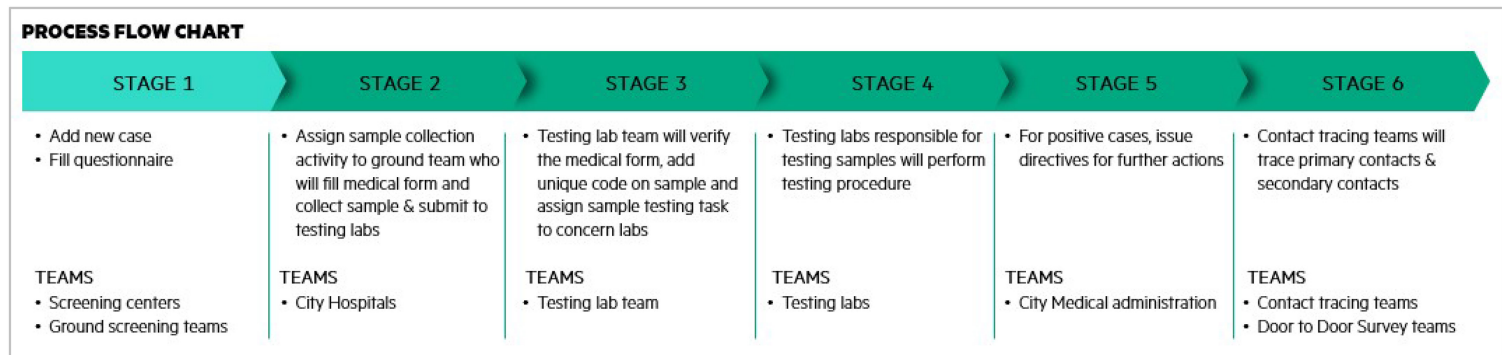

Fig. 6. COVID Management Platform.

Step 2. Test centre allocation for positive cases.

Step 3. Test results management.

Step 4. Treatment centre allocation based on the test result and severity of the symptoms experienced in individual cases.

Step 5. Recording the details of the identified cases and the first and second contacts of the subject for contact tracing.

It consists of integrated modules for:

- Identifying new suspect cases [11]

- Analysing COVID-19 test requirements through a questionnaire module [9, 10, 11]

- Reporting generation through a three-step lab module from a sample collection $[9,10]$

- Provision for multi-agency data input and correlation for quick Management Information System (MIS) and dashboard to current trends and to ascertain hotspots [9, 10]

The workflow of the solution is depicted in the diagram in Figure 6. 
The solution has integrated notifications through mobile Short Messaging Service (SMS) to inform various stakeholders in the process flow. The citizen data captured initially is updated periodically at every stage. Field health care professionals and field authorities can use multiple channels such as mobile apps or web-based forms to create and update entries in the system, thus providing a central repository of all cases in the city along with their travel history, symptoms, test results, and contact history. Using the contact history, authorities can trace primary and secondary contacts of the cases, thus following the chain to assess the spread and devise strategies to break the chain.

A few more initiatives that authors executed in Smart cities along with the authorities are $[8,12]$

- Leveraging the Smart City ICCC as the COVID helpline centre/call centre where critical concerns of citizens were received and resolved through an escalation matrix.

- Smart City conference facilities were leveraged as COVID-19 war rooms for discussion, strategy planning, and decision-making for multiple departments involved in managing the pandemic, thus bringing all concerned under one roof.

- Smart City ICCC infrastructure was designed for remote monitoring of patients by trained doctors through tele-conferencing/video-conferencing, thereby reducing the need for citizens to move out of their homes if they have symptoms, and reducing exposure to both healthcare staff and citizens. The symptoms were assessed remotely, and teams were assigned to visit for samples only if required.

Benefits and Next Steps

Benefits listed below are accrued out of the ICCC extended COVID management system [7-10].

- ICT-enabled civic services support precluded the need for physical presence of citizens for rendering such services

- Need-based outdoor field staff deployment

- Reduced exposure hazards to the COVID-19 warriors

- Remote management with auto incident detection

- System-driven SOP execution from ICCC

- Real-time data assisted decision-making pertaining to quarantine, containment, testing, and COVID-19 care assistance

Smart City infrastructure will provide a coherent convergence point for the stakeholders to evaluate conditions in real time and undertake appropriate actions during normal and emergency situations. Image data captured by city surveillance cameras and applications are instantly used for the sole purpose of executing government promulgated COVID-19 SoP execution and not stored anywhere.

\section{CONCLUSION}

By leveraging both the cyber-physical (IoT) systems and the ICCC in a Smart City, the authors have helped authorities of certain cities combat the COVID-19 pandemic effectively and efficiently [8-11]. By further finetuning the experiences and the lessons learned, the authors feel that the cities that have implemented the above solutions are better positioned to manage such pandemics now and in the future, and can take the lead in advocating other cities on the importance of leveraging cyber-physical systems and a Smart City ICCC for handling pandemics.

\section{ACKNOWLEDGMENTS}

All authors of this article are experienced IT Professionals employed with Hewlett Packard Enterprise India Private Limited in different capacities, engaged in developing, deploying and managing Smart City solutions. 
The opinions and positions presented in this article are those of the individual authors and do not represent the opinion or position of Hewlett Packard Enterprise or any of its affiliates.

\section{REFERENCES}

[1] ICMR COVID Study Group, COVID Epidemiology and Data Management Team, COVID Laboratory Team, and VRDLN Team. Laboratory surveillance for SARS-CoV-2 in India: Performance of testing, descriptive epidemiology of detected COVID-19. January 22-April 30, 2020. Indian fournal of Medical Research 151 (5), 424-437.

[2] Smart City Mission Transform Nation, MoHUA Government of India. Retrieved from http://164.100.161.224/upload/uploadfiles/files/ SmartCityGuidelines(1).pdf.

[3] Lancet Editorial. India under COVID-19 Lockdown. Retrieved from https://www.thelancet.com/action/showPdf?pii=S01406736\%2820\%2930938-7. 395 (April 25, 2020), 1315.

[4] Maanak Gupta, Mahmoud Abdelsalam, and Sudip Mittal. 2020. Enabling and Enforcing Social Distancing Measures Using Smart City and ITS Infrastructures: A COVID-19 Use Case. Cornell University, P 3, Para A (Smart City Crowd), Thane.

[5] Gettleman Jeffrey and Schultz Kai. 2020. Modi Orders 3-Week Total Lockdown for All 1.3 Billion Indians. The New York Times (March 2020). ISSN 0362-4331.

[6] BCG and MoHUA. Retrieved from https://smartnet.niua.org/sites/default/files/resources/indian_smart_cities_-_covid-19_response_ vinal.pdf.

[7] M. Z. M. Nomani, Mohammad Rauf, Zubair Ahmed, Tarique Faiyaz, Saif A. Khan, and Madiha Tahreem. 2020. Quarantine Law Enforcement and Corona Virus (COVID-19) Pandemic in India JXU_Vol_14_4_2020.pdf 14 (4), 536.

[8] Abhishek Raval. Bhopal Emerges as the Hotbed for Innovation, Using Smart Technologies for Managing COVID-19. Retrieved April 12, 2020 from https://www.expresscomputer.in/news/bhopal-emerges-as-the-hotbed-for-innovation-using-smart-technologies-formanaging-covid-19/52824/.

[9] Ravi Pratap Singh, Mohd Javaid, Abid Haleem, and Rajiv Suman. 2020. Internet of Things (IoT) Applications to Fight against COVID-19 Pandemic, Diabetes, and Metabolic Syndrome., Clinical Research and Reviews 14 (4), 521-524.

[10] SCC India Staff. See How Dehradun Smart City is Combating COVID-19. Retrieved May 15, 2020 from https://india.smartcitiescouncil. com/article/see-how-dehradun-smart-city-combating-covid-19.

[11] Strategy of COVID19 Testing in India. 2020. ICMR 2-3 Strategy_COVID19_testing_India.pdf.

[12] Tanushree Venkatraman. Thane, 25 cities adopt smart tech to combat coronavirus. Retrieved July 1, 2020 from https://www. hindustantimes.com/mumbai-news/thane-25-cities-adopt-smart-tech-to-combat-coronavirus/story-9OU9dgwHPuKNJyjNmlI9tL. html.

Received August 2020; revised September 2020; accepted October 2020

Digital Government: Research and Practice, Vol. 2, No. 1, Article 8. Publication date: November 2020. 